\title{
Coulombic effects on magnetoconductivity oscillations induced by microwave excitation in multisubband two-dimensional electron systems
}

\author{
Yu.P. Monarkha ${ }^{1}$ \\ ${ }^{1}$ Institute for Low Temperature Physics and Engineering, 47 Lenin Avenue, 61103 Kharkov, Ukraine
}

\begin{abstract}
We develop a theory of magneto-oscillations in photoconductivity of multisubband twodimensional electron systems which takes into account strong Coulomb interaction between electrons. In the presence of a magnetic field oriented perpendicular, internal electric fields of fluctuational origin cause fast drift velocities of electron orbit centers which affect probabilities of intersubband scattering and the photoconductivity. For the electron system formed on the liquid helium surface, internal forces are shown to suppress the amplitude of magneto-oscillations, and change positions of magnetoconductivity minima which evolve in zero-resistance states for high radiation power.
\end{abstract}

PACS numbers: 73.40.-c,73.20.-r,73.25.+i, 78.70.Gq

\section{INTRODUCTION}

Observation of microwave-resonance-induced magnetoconductivity oscillations in the multisubband twodimensional (2D) electron system formed on the free surface of liquid helium 1,2 have attracted much interest. These oscillations appear to be very similar to $1 / B$-periodic resistivity $\left(R_{x x}\right)$ oscillations observed in ultrahigh-mobility GaAs/AlGaAs heterostructures subjected to a dc magnetic field and to strong microwave (MW) radiation of a quite arbitrary frequency $\omega>\omega_{c} \underline{\underline{3}} \underline{\underline{5}}$ (here $\omega_{c}$ is the cyclotron frequency). At low temperatures $T \simeq 0.2 \mathrm{~K}$, oscillations reported for surface electrons (SEs) on liquid helium have a peculiar shape which is reminiscent of a derivative of a peaky function rather than a sum of simple maxima expected for usual scattering. With an increase in radiation power, the minima of the magnetooscillations evolve in zeromagnetoconductivity $\sigma_{x x}$ states $^{2}$, which are very similar to zero-resistance states (ZRS) observed in semiconductor 2D electron systems $\frac{4,5}{,}$, because, under a strong magnetic field, both $\sigma_{x x}$ and $R_{x x}$ are proportional to the effective collision frequency $\nu(B)$. The only important difference of these two similar phenomena is that for SEs on liquid helium magneto-oscillations are observed only in the vicinity of a specific MW frequency $\omega=\omega_{2,1}$, representing the resonance frequency for excitation of the second surface subband.

In semiconductor systems, ZRS are explained $\underline{\underline{6}}$ as a consequence of the negative linear conductivity condition $\sigma_{x x}<0$ which appears for high radiation power. Due to instability of the system under this condition, it enters a nonlinear regime and develops a steady current state with $\sigma_{x x}\left(j_{0}\right)=0$. Mechanisms of the negative linear response conductivity of a $2 \mathrm{D}$ electron gas formed in semiconductor structures are based on photon-induced impurity scattering within the ground subband ${ }^{7,8}$. They were quite successful in explaining magneto-oscillations and ZRS induced by MW radiation in semiconductor systems. Unfortunately, they cannot be applied for explanation of negative conductivity effects in the $2 \mathrm{D}$ electron system formed on the liquid helium surface, because the MW frequency $\omega$ considered in these theories is not restricted by the condition $\omega=\omega_{2,1}$.

The new mechanism of the negative linear response conductivity reported recently $\stackrel{9,10}{ }$ is based on nonequilibrium filling of the second surface subband,

$$
N_{2}>N_{1} \exp \left(-\hbar \omega_{2,1} / T_{e}\right)
$$

induced by MW excitation (here $N_{l}$ is the number of electrons at the corresponding surface subband and $T_{e}$ is the electron temperature). This condition provides a new channel for negative momentum relaxation due to usual quasi-elastic inter-subband scattering. This possibility can be seen already from the energy conservation for electron scattering from the excited subband $(l=2)$ to the ground subband $(l=1)$.

Consider the electron energy spectrum in a magnetic filed applied perpendicular to the surface

$$
\varepsilon_{l, n, X}=\Delta_{l}+\hbar \omega_{c}(n+1 / 2)-e E_{\|} X
$$

(here $\Delta_{l}$ is the spectrum of SE states, $n=0,1,2 \ldots, X$ is the center coordinate of the cyclotron motion, and $E_{\|}$ is the dc electric field directed antiparallel to the $x$-axis). Then, the energy conservation yields

$$
\hbar \omega_{c}\left(n-n^{\prime}\right)+\hbar \omega_{2,1}-e E_{\|}\left(X-X^{\prime}\right)=0 .
$$

In this equation, $\hbar \omega_{2,1}$ is not a photon quantum. It is the energy difference for electron excitation in the $z$-direction $\left(\hbar \omega_{l, l^{\prime}}=\Delta_{l}-\Delta_{l^{\prime}}\right)$. Since $\omega_{2,1}$ is substantially higher than $\omega_{c}$, and $e E_{\|} L_{B} \ll \hbar \omega_{c}$ (here $L_{B}^{2}=\hbar c / e B$ ), scattering down the surface levels means scattering up the Landau levels $n^{\prime}-n \equiv m^{*}>0$ and we have

$$
e E_{\|}\left(X-X^{\prime}\right)=\hbar \omega_{c}\left(\frac{\omega_{2,1}}{\omega_{c}}-m^{*}\right) .
$$

Thus, quasi-elastic inter-subband scattering from $l=2$ to $l=1$ will be the scattering against the driving force $\left(X^{\prime}<X\right)$, if $\omega_{2,1} / \omega_{c}-m^{*}>0$, or when $B$ is a bit lower than the level matching point $\omega_{2,1} / \omega_{c}=m^{*}$. At 
the same conditions, electron scattering up the surface levels is obviously the scattering along the driving force $\left(X^{\prime}>X\right)$. Therefore, for the appearance of the negative conductivity correction, the additional condition of Eq. (1) is necessary.

In semiconductor $2 \mathrm{D}$ electron systems ${ }^{4}$, resistance minima occur at $\omega / \omega_{c}=m^{*}+1 / 4$. For surface electrons on liquid helium of the areal density $n_{e} \simeq 10^{6} \mathrm{~cm}^{-2}$, magnetoconductivity minima reported in Ref. 2 are also located approximately near these "magic" numbers $m^{*}+$ $1 / 4$. Therefore, it is interesting to investigate if there is really such strong correlation in positions of $R_{x x}$ and $\sigma_{x x}$ minima for these two different phenomena induced by MW excitation. In the single-electron theory of MWinduced magnetooscillations ${ }^{10}$, the conductivity minima are placed substantially closer to the level matching numbers $m^{*}$, because the Landau level broadening is very small for SEs on liquid helium. It is expected that larger distances of $\sigma_{x x}$ minima from the level matching points observed in the experiment will be explained by the many-electron effect. An additional interest in studying this problem is inspired by the observation of the resonant photovoltaic effect which emerges at the minima of conductivity oscillations ${ }^{11}$.

The important conclusion of the single-electron treatment of MW-induced magneto-oscillations is that the negative conductivity terms are large for an electron system with extremely narrow Landau levels, when the collision broadening of Landau levels $\Gamma_{n}$ is much smaller than temperature $T$. This condition is well realized for surface electrons on liquid helium. Still, under usual experimental conditions, this electron system is in the strong Coulomb coupling regime, which means that the average Coulomb interaction energy of an electron $U_{C}$ is much larger than $T$ and $\hbar \omega_{c}$. Therefore, a thorough investigation of Coulombic effects on magnetoconductivity oscillations is required.

In this work, we report the many-electron theory of magnetoconductivity oscillations induced by resonance MW excitation which takes into account strong Coulomb interaction between electrons. We use the fluctuational electric field concept $^{12}$ which was generalized ${ }^{13,14}$ to incorporate the collision broadening of Landau levels. In the new theory, the Coulomb interaction between electrons affects strongly the line-shape and broadening of magnetoconductivity oscillations. As a result, positions of $\sigma_{x x}$ minima become dependent strongly on electron density $n_{e}$ and on the level matching number $m^{*}$. This Coulombic effect appears to be very sensitive to electron temperature, therefore, it could be used for estimation of electron heating in experiments on MW-induced magnetooscillations.

\section{DECAY RATE AND OCCUPANCY OF EXCITED SUBBANDS}

At low enough temperatures, depending on electron density, the 2D electron system formed on the free surface of liquid helium undergoes the Wigner solid transition. This transition occurs when $U_{C} / T \simeq 137^{15}$. Therefore, there is a broad range $1 \ll U_{C} / T<137$, where the electron system represents a highly correlated Coulomb liquid. These conditions are quite usual for experiments on SEs in liquid helium. It is well established that already at $U_{C} / T>9$ the electron velocity autocorrelation time $\tau_{e-e}$ is very high and close to the reciprocal of the harmonic oscillator frequency in a $2 \mathrm{D}$ triangular electron lattice ${ }^{16}$. This allows to simplify the transport theory, using the assumption that SEs are at equilibrium in the moving center-of-mass reference frame. In the semiclassical transport theory, this simplest many-electron effect increases the effective collision frequency of SEs by a numerical factor of the order of 2, which depends on the perpendicular electric field $E_{\perp}$. In the presence of the quantizing magnetic field, another kind of many-electron effect becomes important which can substantially reduce the effective collision frequency as compared to the result of the single-electron treatment.

The influence of Coulomb interaction on electron scattering under magnetic field can be well understood in terms of the quasi-uniform electric field of fluctuational origin, acting on each electron of a $2 \mathrm{D}$ electron liquid. It is assumed that, under the condition $U_{C} \gg T_{e}$, an electron is displaced from an equilibrium position due to thermal fluctuations ${ }^{12}$ similar to an electron in a triangular Wigner lattice. When the magnetic length $L_{B}$ is much smaller than the electron spacing $a$, the restoring electric field of other electrons $\mathbf{E}_{f}^{(i)}$ can be considered as a quasiuniform field, causing a fast drift velocity of the electron orbit center $u_{f}^{(i)}=c E_{f}^{(i)} / B$ directed perpendicular to $\mathbf{E}_{f}^{(i)}$. The fast motion of cyclotron obit centers affects strongly the magnetoconductivity of electrons scattered by helium vapor atoms ${ }^{17}$ and capillary wave quanta (ripplons $)^{14}$. Monte-Carlo simulations ${ }^{18}$ indicate that, in a wide range of the ratio $U_{C} / T_{e} \gg 1$, the distribution of the fluctuational electric field is close to a Gaussian with the width parameter given by $\sqrt{\left\langle E_{f}^{2}\right\rangle} \equiv E_{f}^{(0)} \simeq 3 \sqrt{T_{e}} n_{e}^{3 / 4}$.

It is remarkable that a strong quasi-uniform fluctuational field does not smear off Landau levels, because any uniform field can be eliminated by a proper choice of the reference frame. It is the frame where the cyclotron orbit center is at rest, and the electron spectrum coincides with the usual Landau spectrum. In the laboratory frame, the fluctuational field tilts the Landau levels similar to Eq. (2). We shall generalize the many-electron theory of quantum magnetotransport in 2D Coulomb liquids developed in Ref. 13 and 14 by including in consideration inter-subband scattering. In particular, we shall consider the decay rate of the first excited subband, which deter- 
mines subband occupancies under the MW resonance.

The ZRS of SEs are observed at low temperatures $T \leq$ $0.2 \mathrm{~K}$, where electrons are predominantly scattered by capillary wave quanta. The electron-ripplon interaction is usually described by the following Hamiltonian 14

$$
\begin{gathered}
H_{\mathrm{int}}\left(\mathbf{R}_{e}\right)=\frac{1}{A} \sum_{\mathbf{q}} Q_{q}\left(b_{\mathbf{q}}+b_{-\mathbf{q}}^{\dagger}\right) U_{q}\left(z_{e}\right) e^{i \mathbf{q} \cdot \mathbf{r}_{e}}, \\
U_{q}(z)=\Lambda q^{2} W(q z)+e E_{\perp}-\frac{\partial V_{e}^{(0)}}{\partial z_{e}}, \\
W(y)=\frac{1}{y^{2}}-\frac{K_{1}(y)}{y},
\end{gathered}
$$

where $A$ is the surface area, $Q_{q}=\sqrt{\hbar q / 2 \rho \omega_{q}}, \omega_{q} \simeq$ $\sqrt{\alpha / \rho} q^{3 / 2}$ is the ripplon spectrum, $\mathbf{R}_{e}=\left\{z_{e}, \mathbf{r}_{e}\right\}, \hbar \mathbf{q}$ is the ripplon momentum, $b_{-\mathbf{q}}^{\dagger}$ and $b_{\mathbf{q}}$ are the creation and destruction operators, $\Lambda=e^{2}(\epsilon-1) / 4(\epsilon+1), \epsilon$ is the liquid helium dielectric constant, and $K_{1}(x)$ is the modified Bessel function of the second kind.

In the presence of the perpendicular electric field $E_{\perp}$, the wave functions of the first two surface subbands can be approximated by

$$
\varphi_{1}(z)=A_{1} z e^{-\gamma_{1} z}, \varphi_{2}(z)=A_{2} z\left[1-\frac{2 \bar{\gamma}_{2,1}}{3} z\right] e^{-\gamma_{2} z},
$$

where $A_{l}$ are normalization constants, parameters $\gamma_{l}$ are found by the variation, and $\bar{\gamma}_{l, l^{\prime}}=\left(\gamma_{l}+\gamma_{l^{\prime}}\right) / 2$. In the magnetotransport theory, using the dimensionless parameter $x_{q}=q^{2} L_{B}^{2} / 2$, it is convenient to represent matrix elements of Eq. (6) as $\left(U_{q}\right)_{l, l^{\prime}}=\Lambda V_{l, l^{\prime}}\left(x_{q}\right) / L_{B}^{2}$, where

$$
\begin{gathered}
V_{l, l^{\prime}}(x)=x w_{l, l^{\prime}}\left(\frac{x}{2 \bar{\gamma}_{l, l^{\prime}}^{2} L_{B}^{2}}\right)+\frac{F_{l, l^{\prime}} L_{B}^{2}}{\Lambda}, \\
F_{l, l^{\prime}}=e E_{\perp} \delta_{l, l^{\prime}}+\left(\frac{\partial \mathrm{v}}{\partial z}\right)_{l, l}^{1 / 2}\left(\frac{\partial \mathrm{v}}{\partial z}\right)_{l^{\prime}, l^{\prime}}^{1 / 2}-\left(\frac{\partial \mathrm{v}}{\partial z}\right)_{l, l^{\prime}},
\end{gathered}
$$

$\mathrm{v}(z)=-\Lambda / z+e E_{\perp} z$, and the functions $w_{l, l^{\prime}}(y)$ represent the matrix elements of $2 W(q z)$. Useful expressions for the dimensionless functions $w_{l, l^{\prime}}(y)$ are given in the Appendix.

At first, consider the probability of inter-subband scattering of an electron moving in crossed magnetic $\mathbf{B}$ and electric $\mathbf{E}_{\|}$fields. The matrix elements of the interaction Hamiltonian yield $X^{\prime}-X=-q_{y} L_{B}^{2}$, therefore, the term $e E_{\|}\left(X-X^{\prime}\right)$ entering the energy conservation of Eq. (3) can be rewritten as $\hbar \mathbf{q} \cdot \mathbf{u}_{d}$, where $\mathbf{u}_{d}$ is the drift velocity $\left(u_{d}=c E_{\|} / B\right)$. Then, in the Born approximation, rates of inter-subband scattering can be found as

$$
\nu_{l, n \rightarrow l^{\prime}, n^{\prime}}=\frac{2 \pi}{\hbar} \sum_{\mathbf{q}} Q_{q}^{2}\left(2 N_{q}+1\right)\left|\left(U_{q}\right)_{l, l^{\prime}}\right|^{2} \times
$$

$$
\times J_{n, n^{\prime}}^{2}\left(x_{q}\right) \delta\left(\varepsilon_{n^{\prime}}-\varepsilon_{n}+\hbar \mathbf{q} \cdot \mathbf{u}_{d}+\Delta_{l^{\prime}, l}\right),
$$

where $\Delta_{l, l^{\prime}}=\Delta_{l}-\Delta_{l^{\prime}}$,

$$
J_{n, n^{\prime}}^{2}(x)=\frac{\left[\min \left(n, n^{\prime}\right)\right] !}{\left[\max \left(n, n^{\prime}\right)\right] !} x^{\left|n-n^{\prime}\right|} e^{-x}\left[L_{\min \left(n, n^{\prime}\right)}^{\left|n-n^{\prime}\right|}(x)\right]^{2},
$$

$L_{n}^{m}(x)$ are the associated Laguerre polynomials, and $N_{q}$ is the ripplon distribution function.

Since Eq. (10) is independent of $X$, one can find the average rate $\bar{\nu}_{l \rightarrow l^{\prime}}$ using equilibrium electron distribution over Landau levels: $Z_{\|}^{-1} e^{-\varepsilon_{n} / T_{e}}$, where $Z_{\|}=$ $\sum_{n} e^{-\varepsilon_{n} / T_{e}}$, and $\varepsilon_{n}$ is the Landau spectrum. For an ensemble of electrons, this procedure is equivalent to the assumption that electrons are in equilibrium in the moving center-of-mass reference frame, where $\mathbf{E}_{\|}^{\prime}=0$. In order to include the collision broadening of Landau levels, we follow Ref. 19 and represent the probability of scattering in terms of density-of-state functions of the initial and final states. Then, $\delta\left(\varepsilon-\varepsilon_{n}\right)$ of the density-of-state functions can be replaced by $-\frac{1}{\pi \hbar} \operatorname{Im} G_{l, n}(\varepsilon)$. Here $G_{l, n}(\varepsilon)$ is the single-electron Green's function of the corresponding subband, whose imaginary part is broadened due to collisions with scatterers. This procedure is equivalent to the self-consistent Born approximation (SCBA) ${ }^{20}$.

Using the Gaussian shape of Landau level densities, inter-subband scattering rates can be represented as

$$
\bar{\nu}_{l \rightarrow l^{\prime}}=\frac{\hbar}{m_{e} A} \sum_{\mathbf{q}} \chi_{l, l^{\prime}}(q) S_{l, l^{\prime}}^{(0)}\left(q, \omega_{l, l^{\prime}}-\mathbf{q} \cdot \mathbf{u}_{d}\right)
$$

where $m_{e}$ is the free electron mass,

$$
\begin{gathered}
\chi_{l, l^{\prime}} \simeq \frac{m_{e} T L_{B}^{2}}{2 \alpha \hbar^{3} x_{q}}\left|\left(U_{q}\right)_{l, l^{\prime}}\right|^{2}, \\
S_{l, l^{\prime}}^{(0)}(q, \omega)=\frac{2 \pi^{1 / 2} \hbar}{Z_{\|}} \sum_{n, n^{\prime}} \frac{J_{n, n^{\prime}}^{2}\left(x_{q}\right)}{\Gamma_{l, n ; l^{\prime}, n^{\prime}}} e^{-\varepsilon_{n} / T_{e}} \times \\
\times \exp \left[-\left(\frac{\hbar \omega-m \hbar \omega_{c}-\Gamma_{l, n}^{2} / 4 T_{e}}{\Gamma_{l, n ; l^{\prime}, n^{\prime}}}\right)^{2}+\frac{\Gamma_{l, n}^{2}}{8 T_{e}^{2}}\right],
\end{gathered}
$$

$m=n^{\prime}-n, \Gamma_{l, n}$ is the collision broadening of Landau levels of the corresponding subband, and $2 \Gamma_{l, n ; l^{\prime}, n^{\prime}}^{2}=$ $\Gamma_{l, n}^{2}+\Gamma_{l^{\prime}, n^{\prime}}^{2}$. In Eq. (12), we consider $N_{q} \simeq T / \hbar \omega_{q} \gg 1$. For short-range scatterers, the collision broadening is independent of $n\left(\Gamma=\hbar \sqrt{2 \omega_{c} \nu / \pi}\right.$, here $\nu$ is the collision frequency in the absence of the magnetic field). In the case of electron-ripplon interaction, the collision broadening of Landau levels depends on $n^{14}$. At $\mathbf{q} \cdot \mathbf{u}_{d} \rightarrow 0$, Eq. (11) coincides with the decay rate found previously in the absence of $\mathbf{E}_{\|}^{10,21}$.

For $l=l^{\prime}$, the factor $S_{l, l^{\prime}}^{(0)}(q, \omega)$ represents the dynamical structure factor of a nondegenerate $2 \mathrm{D}$ system of noninteracting electrons. The definition of Eq. (13) extends 
the factor for $l \neq l^{\prime}$ to describe inter-subband scattering. Relationship $S_{l, l^{\prime}}(\mathbf{q}, \omega)=S_{l, l^{\prime}}^{(0)}\left(q, \omega-\mathbf{q} \cdot \mathbf{u}_{d}\right)$, which follows from Eq. (11), represents the Galilean invariance along the interface for the generalized factor, assuming electrons are in equilibrium in the center-of-mass reference frame, where $\mathbf{E}_{\|}^{\prime}=0$.

The Eq. (11) describes how a fast drift velocity affects the probability of inter-subband scattering. As noted above, the Coulomb liquid can be approximately considered as an ensemble of independent electrons whose orbit centers move fast $\left(\mathbf{u}_{d} \rightarrow \mathbf{u}_{f}^{(i)}\right)$ due to the fluctuational electric field $\mathbf{E}_{f}^{(i)}$. In experiments on MWinduced magneto-oscillations of surface electrons in liquid helium, typical electron spacing $a$ varies approximately from $1 \cdot 10^{-3} \mathrm{~cm}$ to $3 \cdot 10^{-4} \mathrm{~cm}$, which is much larger than the typical electron localization radius in the perpendicular direction $\gamma_{1}^{-1} \sim 10^{-6} \mathrm{~cm}$. Therefore, we can assume that the fluctuational electric field acting on electrons, occupying first few excited subbands, is the same as that acting on electrons of the ground subband.

In the single-electron theory ${ }^{10}$, scattering probabilities and the momentum relaxation rate of a multisubband $2 \mathrm{D}$ electron system are found in terms of the generalized dynamic factor $S_{l, l^{\prime}}^{(0)}(q, \omega)$ using its equilibrium properties. Therefore, the main goal of the many-electron theory is to construct the Coulomb liquid version of this factor $S_{l, l^{\prime}}^{(\mathrm{me})}(q, \omega)$ applicable under conditions $U_{C} / T \gg 1$. Following the ideas developed for intra-subband scattering, we expect that the many-electron dynamical factor of the multi-subband system of strongly interacting electrons, can be approximated as

$$
S_{l, l^{\prime}}^{(\mathrm{me})}(q, \omega) \simeq\left\langle S_{l, l^{\prime}}^{(0)}\left(q, \omega-\mathbf{q} \cdot \mathbf{u}_{f}\right)\right\rangle_{f}
$$

where $\langle\ldots\rangle_{f}$ denotes averaging over the fluctuational electric field. For $l=l^{\prime}$, this relationship was already proven 13,14 . For inter-subband scattering, the approximation of Eq. (14) follows from the simple averaging of Eq. (11) over the fluctuational electric field, where $\mathbf{u}_{f}$ substitutes for $\mathbf{u}_{d}$. Since the maxima of $S_{l, l^{\prime}}^{(0)}(q, \omega)$ as a function of frequency has a simple Gaussian shape, the model of Eq. (14) provides larger broadening of these $\operatorname{maxima} \sqrt{\Gamma_{l, n ; l^{\prime}, n^{\prime}}^{2}+x_{q} \Gamma_{C}^{2}}$ (here $\left.\Gamma_{C}=\sqrt{2} e E_{f}^{(0)} L_{B}\right)$, which agrees with the broadening of the dynamical structure factor of the 2D Wigner solid under a strong magnetic field ${ }^{22}$.

When considering inter-subband scattering, we must separate scattering down and up the surface subbands. For electron scattering down $\left(l>l^{\prime}\right)$, at typical electron densities $n_{e} \sim 10^{6} \mathrm{~cm}^{-2}$, the approximation of Eq. (14) is quite sufficient. For description of electron scattering up the surface subbands, an equilibrium form of $S_{l, l^{\prime}}^{(\mathrm{me})}(q, \omega)$ should contain also small frequency shifts which provide the detailed balancing $\bar{\nu}_{l^{\prime} \rightarrow l}=\bar{\nu}_{l \rightarrow l^{\prime}} \exp \left(-\hbar \omega_{l, l^{\prime}} / T_{e}\right)$. Correct frequency shifts of $S_{l, l^{\prime}}^{(\mathrm{me})}(q, \omega)$ can be obtained from the detailed balancing, or using an accurate model based on properties of the 2D Wigner solid under a strong magnetic field ${ }^{14,22}$. This approach yields

$$
\begin{aligned}
S_{l, l^{\prime}}^{(\mathrm{me})}(q, \omega) & =\frac{2 \pi^{1 / 2} \hbar}{Z_{\|}} \sum_{n, n^{\prime}} \frac{J_{n, n^{\prime}}^{2}\left(x_{q}\right)}{\sqrt{\Gamma_{l, n ; l^{\prime}, n^{\prime}}^{2}+x_{q} \Gamma_{C}^{2}}} \times \\
& \times e^{-\varepsilon_{n} / T_{e}} I_{l, n ; l^{\prime}, n^{\prime}}^{(\mathrm{me})}(\omega),
\end{aligned}
$$

where

$$
\begin{gathered}
I_{l, n ; l^{\prime}, n^{\prime}}^{(\mathrm{me})}(\omega)=\exp \left\{-D_{l, n ; l^{\prime}, n^{\prime}}(\omega)\right\} \\
D_{l, n ; l^{\prime}, n^{\prime}}(\omega)=\frac{\left(\hbar \omega-m \hbar \omega_{c}-\frac{\Gamma_{l, n}^{2}}{4 T_{e}}-\frac{x_{q} \Gamma_{C}^{2}}{4 T_{e}}\right)^{2}}{\Gamma_{l, n ; l^{\prime}, n^{\prime}}^{2}+x_{q} \Gamma_{C}^{2}}-\frac{\Gamma_{l, n}^{2}}{8 T_{e}^{2}},
\end{gathered}
$$

and $m=n^{\prime}-n$. Frequency shifts $\Gamma_{l, n}^{2} / 4 T_{e}$ and $x_{q} \Gamma_{C}^{2} / 4 T_{e}$ are small as compared to $\hbar \omega_{c}$. It should be noted that the approximation of Eq. (14) gives Eqs. (15) and (16) without the small frequency shift $x_{q} \Gamma_{C}^{2} / 4 T_{e}$. We restored it from the expression for the dynamical structure factor of the Wigner solid. This shift follows also from the detailed balancing, because it provides the important property of the equilibrium factor

$$
S_{l, l^{\prime}}^{(\mathrm{me})}(q,-\omega)=e^{-\hbar \omega / T_{e}} S_{l^{\prime}, l}^{(\mathrm{me})}(q, \omega),
$$

which is equivalent to the detailed balancing.

Thus, to describe inter-subband scattering rates $\bar{\nu}_{l \rightarrow l^{\prime}}$ in the multi-subband Coulomb liquid, one can use the general equation similar to Eq. (11), where the equilibrium many-electron factor $S_{l, l^{\prime}}^{(\mathrm{me})}\left(q, \omega_{l, l^{\prime}}\right)$ of Eq. (15) substitutes for $S_{l, l^{\prime}}^{(0)}\left(q, \omega_{l, l^{\prime}}-\mathbf{q} \cdot \mathbf{u}_{d}\right)$. The Coulomb broadening of the generalized factor $\sqrt{x_{q}} \Gamma_{C}$ is not equivalent to the collision broadening of the SCBA theory, because it depends on the wave-vector argument of $S_{l, l^{\prime}}^{(\mathrm{me})}(q, \omega)$, which complicates evaluations.

Consider the decay rate of the first excited surface subband $\bar{\nu}_{2 \rightarrow 1}$. The usage of Eqs. (15) and (16) yields

$$
\begin{gathered}
\bar{\nu}_{2 \rightarrow 1}=\frac{\Lambda^{2} T}{2 \sqrt{\pi} \alpha \hbar L_{B}^{4} Z_{\|}} \sum_{n, m} \frac{n !}{(n+m) !} e^{-\varepsilon_{n} / T_{e}} \times \\
\times \int_{0}^{\infty} V_{2,1}^{2}(x) \frac{x^{m-1}\left[L_{n}^{m}(x)\right]^{2}}{\sqrt{\Gamma_{2, n ; 1, n+m}^{2}+x \Gamma_{C}^{2}}} \times \\
\times \exp \left[-x-\frac{\hbar^{2}\left(\omega_{2,1}-m \omega_{c}-x \Gamma_{C}^{2} / 4 \hbar T_{e}\right)^{2}}{\Gamma_{2, n ; 1, n+m}^{2}+x \Gamma_{C}^{2}}\right] d x,
\end{gathered}
$$

where $m$ is a positive integer, and the frequency shift $\Gamma_{l, n}^{2} / 4 T_{e}$ is disregarded as well as the small parameter $\Gamma_{l, n}^{2} / 8 T_{e}^{2}$. Here we still keep the Coulomb shift 


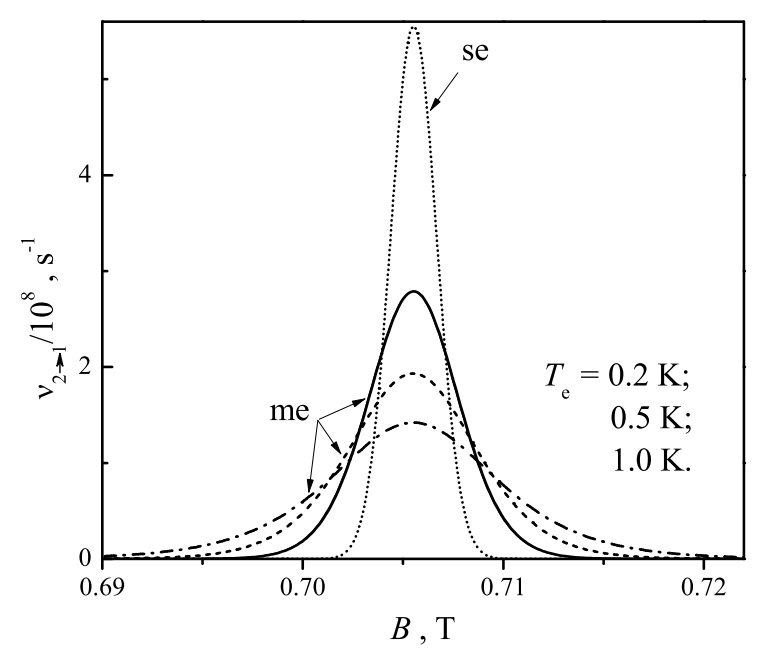

FIG. 1. Decay rate of the first excited subband $\nu_{2} \rightarrow 1$ vs the magnetic field $B$ for $T=0.2 \mathrm{~K}$ and $n_{e}=10^{6} \mathrm{~cm}^{-2}$ : singleelectron treatment (dotted); many-electron theory $\left[T_{e}=T\right.$ (solid), $T_{e}=0.5 \mathrm{~K}$ (dashed), and $T_{e}=1 \mathrm{~K}$ (dash-dotted)].

$x_{q} \Gamma_{C}^{2} / 4 \hbar T_{e}$ because it can be important at high electron densities.

For typical conditions of experiments 1,2 performed on SEs over liquid ${ }^{3} \mathrm{He}$, the results of numerical evaluation of Eq. (18) are shown in Fig. 1. The magnetic field range is chosen to be a vicinity of the level matching number $m^{*}=4$. One can see that already at a very low electron density $n_{e}=10^{6} \mathrm{~cm}^{-2}$, the many-electron curve calculated for $T_{e}=T=0.2 \mathrm{~K}$ (solid line) substantially broader than the result given by the singleelectron theory (dotted line). Under these conditions $U_{C} / T \simeq 15$. The broadening of the many-electron curve increases strongly with $n_{e}$ and the level matching number $m^{*}$ due to $\Gamma_{C} \propto n_{e}^{3 / 4} / B^{1 / 2}$. Another important conclusion, which follows from this figure, is that the many-electron curve becomes even broader with heating of electrons because $\Gamma_{C} \propto \sqrt{T_{e}}$. It should be noted also that the single-electron decay rate has much weaker dependence on $T_{e}$ which is not shown in Fig. 1.

The above noted Coulomb broadening of the decay rate $\bar{\nu}_{2 \rightarrow 1}$ affects strongly subband occupancies $\bar{n}_{l}=N_{l} / N$ in the presence of MW radiation. For the two-subband model, which is valid for $T_{e} \leqslant 2 K$, we have

$$
\frac{\bar{n}_{2}}{\bar{n}_{1}}=\frac{1+\exp \left(-\hbar \omega_{2,1} / T_{e}\right) \bar{\nu}_{2 \rightarrow 1} / r_{\mathrm{mw}}}{1+\bar{\nu}_{2 \rightarrow 1} / r_{\mathrm{mw}}}
$$

where $r_{\mathrm{mw}}$ is the MW excitation rate. Under the resonance condition, $r_{\mathrm{mw}}=\Omega_{R}^{2} / 2 \gamma_{\mathrm{mw}}$, where $\gamma_{\mathrm{mw}}$ is the halfwidth of the resonance, and $\Omega_{R}$ is the Rabi frequency proportional to the amplitude of the MW field. Thus, the fluctuational electric field leads to much broader variations of $\bar{n}_{2}(B)$ near the level matching condition $\omega_{2,1} / \omega_{c}=m^{*}$ than those given by the single-electron

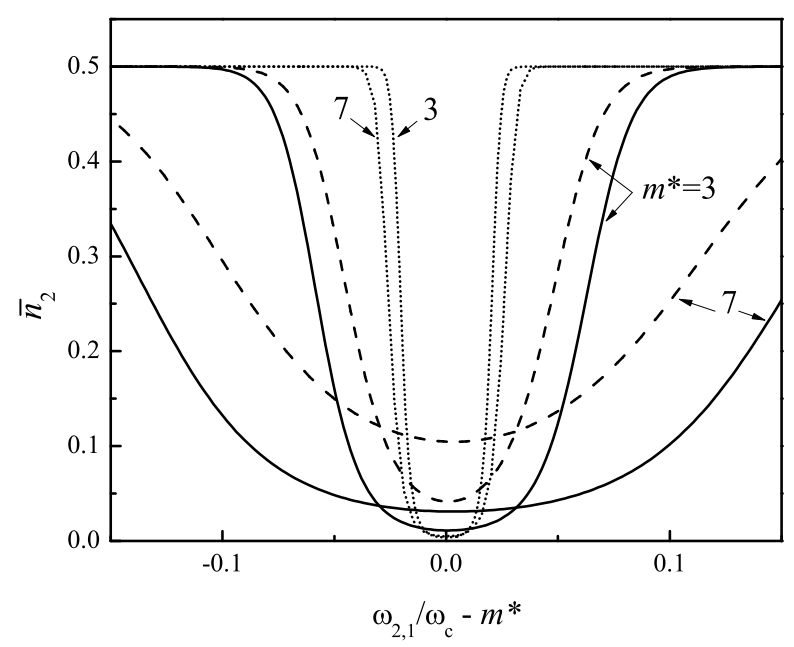

FIG. 2. The occupancy of the first excited subband vs the parameter $\omega_{2,1} / \omega_{c}-m^{*}$ for $T=0.2 \mathrm{~K}, n_{e}=2 \cdot 10^{6} \mathrm{~cm}^{-2}$ : single-electron treatment (dotted), and many-electron theory $\left[\Omega_{R}=2.5 \cdot 10^{7} \mathrm{~s}^{-1}\right.$ (solid), and $\Omega_{R}=5 \cdot 10^{7} \mathrm{~s}^{-1}$ (dashed).

theory.

For electron density $n_{e}=2 \cdot 10^{6} \mathrm{~cm}^{-2}$ and $T_{e}=$ $T=0.2 \mathrm{~K}$, variations of the fractional occupancy $\bar{n}_{2}$ are shown in Fig. 2 When conducting numerical evaluations, we assume that the width of the MW resonance is due to inhomogeneous broadening $2 \gamma_{\mathrm{mw}}=0.3 \mathrm{GHz}^{2}$, and the MW excitation rate $r_{\mathrm{mw}}$ is independent of $B$. The many-electron lines calculated for $m^{*}=3$ and 7 at $\Omega_{R}=2.5 \cdot 10^{7} \mathrm{~s}^{-1}$ (solid) are substantially broader than the corresponding single-electron lines (dotted). The increase of $\bar{n}_{2}$ with MW power is shown by many-electron lines (dashed) calculated at $\Omega_{R}=5 \cdot 10^{7} \mathrm{~s}^{-1}$. Since the negative conductivity correction is proportional to $\bar{n}_{2}-\bar{n}_{1} \exp \left(-\hbar \omega_{2,1} / T_{e}\right)$, it is clear that the many-electron effect should displace strongly conductivity minima.

\section{LINEAR MAGNETOCONDUCTIVITY}

According to the analysis given in the previous Section, probabilities of electron scattering in a multi-subband Coulomb liquid $\left(U_{C} / T>10\right)$ can be described by the many-electron dynamic factor $S_{l, l^{\prime}}^{(\mathrm{me})}(q, \omega)$ of Eq. (15) instead of $S_{l, l^{\prime}}^{(0)}(q, \omega)$ of the single-electron theory. Therefore, using equilibrium properties of $S_{l, l^{\prime}}^{(\mathrm{me})}(q, \omega)$, like that given in Eq. (17), and following the formal procedure described in details in Ref. 10, one can obtain an equation for the momentum relaxation rate similar to that found in the single-electron treatment. The effective collision frequency $\nu$ is a sum of contributions from intrasubband $\left(\nu_{\text {intra }}\right)$ and inter-subband $\left(\nu_{\text {inter }}\right)$ scattering. The $\nu_{\text {inter }}$ consists of normal terms $\left(\nu_{\mathrm{N}}\right)$, existing even at 
$\bar{n}_{l}=\bar{n}_{l^{\prime}} \exp \left(-\hbar \omega_{l, l^{\prime}} / T_{e}\right)$, and anomalous terms $\nu_{\mathrm{A}}$ proportional to $\bar{n}_{l}-\bar{n}_{l^{\prime}} \exp \left(-\hbar \omega_{l, l^{\prime}} / T_{e}\right)$. Simple replacement $S_{l, l^{\prime}}^{(0)}(q, \omega) \rightarrow S_{l, l^{\prime}}^{(\mathrm{me})}(q, \omega)$ yields

$$
\begin{gathered}
\nu_{\text {intra }}=\frac{\hbar \omega_{c}^{2}}{4 \pi T_{e}} \sum_{l} \bar{n}_{l} \int_{0}^{\infty} x_{q} \chi_{l, l}(q) S_{l, l}^{(\mathrm{me})}(q, 0) d x_{q}, \\
\nu_{\mathrm{N}}=\frac{\hbar \omega_{c}^{2}}{4 \pi T_{e}} \sum_{l>l^{\prime}}\left(\bar{n}_{l}+\bar{n}_{l^{\prime}} e^{-\Delta_{l, l^{\prime}} / T_{e}}\right) \times \\
\times \int_{0}^{\infty} x_{q} \chi_{l, l^{\prime}}(q) S_{l, l^{\prime}}^{(\mathrm{me})}\left(q, \omega_{l, l^{\prime}}\right) d x_{q}, \\
\nu_{\mathrm{A}}=-\frac{\omega_{c}^{2}}{4 \pi} \sum_{l>l^{\prime}}\left[\bar{n}_{l}-\bar{n}_{l^{\prime}} e^{-\Delta_{l, l^{\prime}} / T_{e}}\right] \times \\
\quad \times \int_{0}^{\infty} x_{q} \chi_{l, l^{\prime}}(q) \Psi_{l, l^{\prime}}\left(x_{q}\right) d x_{q},
\end{gathered}
$$

were

$$
\Psi_{l, l^{\prime}}\left(x_{q}\right)=-S_{l, l^{\prime}}^{(\mathrm{me})}\left(q, \omega_{l, l^{\prime}}\right)+e^{\Delta_{l, l^{\prime}} / T_{e}} S_{l^{\prime}, l}^{(\mathrm{me})}\left(q,-\omega_{l, l^{\prime}}\right),
$$

and $S_{l, l^{\prime}}^{(\mathrm{me})}(q, \omega) \equiv \partial S_{l, l^{\prime}}^{(\mathrm{me})}(q, \omega) / \partial \omega$. The expression for $\nu_{\mathrm{A}}$ was represented in the form of Eq. (22), containing terms with $l>l^{\prime}$ only, by interchanging the running indices. The similar procedure resulted in Eq. (21) employs the property of Eq. (17).

Using the relationship

$S_{l^{\prime}, l}^{\prime(\mathrm{me})}(q,-\omega)=-e^{-\frac{\hbar \omega}{T_{e}}} S_{l, l^{\prime}}^{(\mathrm{me})}(q, \omega)+\frac{\hbar}{T_{e}} e^{-\frac{\hbar \omega}{T_{e}}} S_{l, l^{\prime}}^{(\mathrm{me})}(q, \omega)$,

which follows from Eqs. (15)-(17), the expression for $\Psi_{l, l^{\prime}}\left(x_{q}\right)$ can be rearranged to a more convenient form:

$$
\begin{aligned}
& \Psi_{l, l^{\prime}}\left(x_{q}\right)=\frac{8 \pi^{1 / 2} \hbar^{2}}{Z_{\|}} \sum_{n, n^{\prime}} \frac{\left|J_{n, n^{\prime}}\left(x_{q}\right)\right|^{2}}{\sqrt{\Gamma_{l, n ; l^{\prime}, n^{\prime}}^{2}+x_{q} \Gamma_{C}^{2}}} e^{-\varepsilon_{n} / T_{e}} \times \\
& \times I_{l, n, l^{\prime} ; n^{\prime}}^{(\mathrm{me}}\left(\omega_{l, l^{\prime}}\right) \frac{\hbar\left(\omega_{l, l^{\prime}}-m \omega_{c}\right)+\left(\Gamma_{l^{\prime}, n^{\prime}}^{2}-\Gamma_{l, n}^{2}\right) / 8 T_{e}}{\Gamma_{l, n ; l^{\prime}, n^{\prime}}^{2}+x_{q} \Gamma_{C}^{2}},
\end{aligned}
$$

where $m=n^{\prime}-n$. It is important that the Coulomb shift $x_{q} \Gamma_{C}^{2} / T_{e}$ does not enter the numerator of Eq. (25), where $\left(\Gamma_{l^{\prime}, n^{\prime}}^{2}-\Gamma_{l, n}^{2}\right) / 8 T_{e}$ can be disregarded for extremely narrow Landau levels. Therefore, the vanishing point of each term entering $\nu_{\mathrm{A}}(B)$ is unaffected by the many-electron effect and practically coincides with the level matching condition $\omega_{2,1}=\left(n^{\prime}-n\right) \omega_{c}$. At the same time, positions of minima and maxima of $\nu_{\mathrm{A}}(B)$ are very sensitive

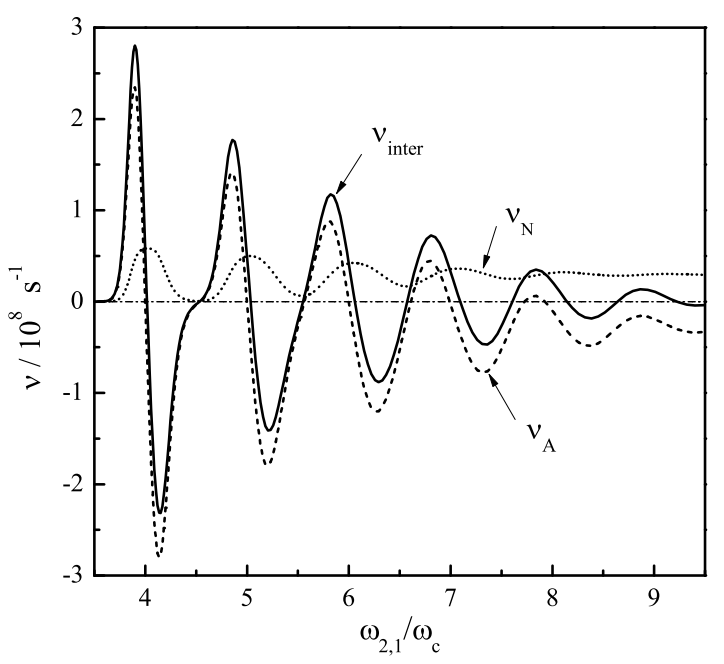

FIG. 3. The effective collision frequency due to inter-subband scattering $\nu_{\text {inter }}$ vs the parameter $\omega_{2,1} / \omega_{c}$ (solid). Two contributions $\nu_{\mathrm{N}}$ and $\nu_{\mathrm{A}}$ are shows by dotted and dashed lines respectively.

to electron density due to the broadening of the generalized dynamic factor $\sqrt{\Gamma_{l, n ; l^{\prime}, n^{\prime}}^{2}+x_{q} \Gamma_{C}^{2}}$. Additionally, at high $n_{e}$, they are slightly affected by the frequency shift $x_{q} \Gamma_{C}^{2} / 4 T_{e}$ entering $I_{l, n, l^{\prime} ; n^{\prime}}^{(\mathrm{me}}\left(\omega_{l, l^{\prime}}\right)$.

Typical behavior of $\nu_{\text {inter }}$ and its components $\left(\nu_{\mathrm{A}}\right.$ and $\left.\nu_{\mathrm{N}}\right)$ as functions of the parameter $\omega_{2,1} / \omega_{c}$ is shown in Fig. 3 for sufficiently high electron density $\left(n_{e}=1.5\right.$. $\left.10^{7} \mathrm{~cm}^{-2}, \Omega_{R}=5 \cdot 10^{7} \mathrm{~s}^{-1}\right)$, when maxima of $S_{l, l^{\prime}}^{(\mathrm{me})}(q, \omega)$ begin to overlap due to the Coulomb broadening $\sqrt{x_{q}} \Gamma_{C}$. Oscillations of $\nu_{\mathrm{A}}(B)$ and $\nu_{\mathrm{N}}(B)$ are quite different. The normal contribution $\nu_{\mathrm{N}}$ is just a sum of simple peaks centered at the level matching conditions $\omega_{2,1}=m^{*} \omega_{c}$. With an increase of the parameter $\omega_{2,1} / \omega_{c}$, maxima overlap strongly, and eventually the dependence $\nu_{\mathrm{N}}(B)$ acquires a positive background.

The sign-changing correction $\nu_{\mathrm{A}}$ originates from the derivative $S_{l, l^{\prime}}^{(\mathrm{me})}(q, \omega)$, and, in the vicinity of the level matching conditions, it is close to zero, if overlapping is small. At larger $m^{*}$, the overlapping of maxima of $I_{l, n, l^{\prime} ; n^{\prime}}^{(\mathrm{me})}\left(\omega_{l, l^{\prime}}\right)$ results in a negative background of $\nu_{\mathrm{A}}(B)$, because the amplitude of $\nu_{\mathrm{A}}$ oscillations decreases with $m^{*}$, and, at a fixed $B$, the negative contribution of smaller $m^{*}$ dominates. It is remarkable that the negative background of $\nu_{\mathrm{A}}(B)$ is compensated by the positive background of $\nu_{\mathrm{N}}(B)$ and the entire contribution from inter-subband scattering $\nu_{\text {inter }}=\nu_{\mathrm{N}}+\nu_{\mathrm{A}}$ (solid line) oscillates nearly with the zero background.

The conductivity equation, which follows from the momentum-balance equation method $\underline{14}$, has the usual Drude form, where, instead of the quasiclassical collision frequency $\nu_{0}$ independent of $B$, it is necessary to use the effective collision frequency $\nu=\nu_{\text {intra }}+\nu_{\mathrm{N}}+\nu_{\mathrm{A}}$. There- 


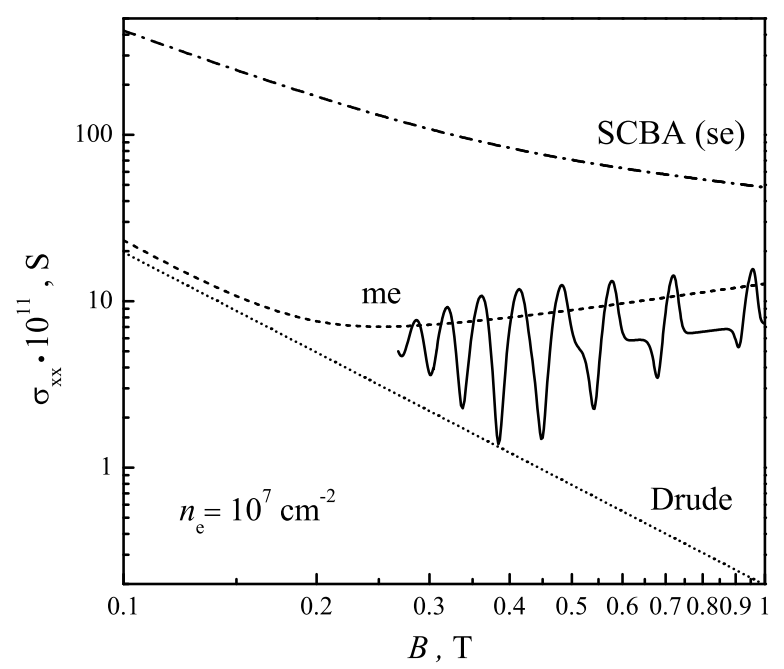

FIG. 4. Magnetoconductivity vs $B$ for $T=0.2 \mathrm{~K}$, and $n_{e}=10^{7} \mathrm{~cm}^{-2}$ : the single-electron treatment based on the SCBA (dash-dotted), the Drude approximation (dotted), and the many-electron theory (dashed). The result obtained for resonant MW excitation with $\Omega_{R}=3.5 \cdot 10^{7} \mathrm{~s}^{-1}$ is shown by the solid line.

fore, under strong magnetic fields, $\sigma_{x x}$ is proportional to $\nu_{\text {intra }}+\nu_{\mathrm{N}}+\nu_{\mathrm{A}}$. Without MW radiation, the influence of the strong Coulomb interaction on $\sigma_{x x}$ is illustrated

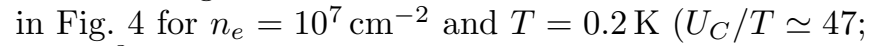
liquid $\left.{ }^{3} \mathrm{He}\right)$. The result of the single-electron treatment based on the SCBA theory (dash-dotted line) was obtained from Eq. (20) by setting $\bar{n}_{1} \rightarrow 1$ and $\Gamma_{C} \rightarrow 0$. When lowering the magnetic field, the many-electron line (dashed) moves from the SCBA line to the Drude line ( $\nu \rightarrow \nu_{0}$, dotted line). Transition to the Drude line occurs due to the Coulomb broadening of $I_{1, n ; 1, n^{\prime}}^{(\mathrm{me})}(0)$ which triggers off scattering processes with $\left|n^{\prime}-n\right|>0$. The many-electron line of Fig. 4 takes into account terms with $\left|n^{\prime}-n\right| \leq 9$.

In the presence of MW radiation, typical behavior of $\sigma_{x x}(B)$ is shown in Fig. 4 by the solid line calculated for $\Omega_{R}=3.5 \cdot 10^{7} \mathrm{~s}^{-1}, n_{e}=10^{7} \mathrm{~cm}^{-2}$, and for a restricted field range covering the level-matching numbers $3 \leq m^{*} \leq 9$. This line illustrates evolution of oscillations and conductivity minima with lowering $B$. In contrast to the single-electron theory, where the amplitude of conductivity oscillations increases with $m^{*}$ (lowering $B)^{9,10}$, here the amplitude of oscillations decreases with $m^{*}$ for sufficiently low magnetic fields, due to the Coulomb broadening of $I_{2, n, 1 ; n+m^{*}}^{(\mathrm{me})}\left(\omega_{2,1}\right)$. In accordance with Fig. 3, conductivity minima of Fig. 4 are quite distant from the level matching points.

The dependence of positions of conductivity minima on the level matching number $m^{*}$ is illustrated in Fig. 5 for lower density $n_{e}=10^{6} \mathrm{~cm}^{-2}$ and two MW powers. This figure indicates that the distance of conductivity ex-

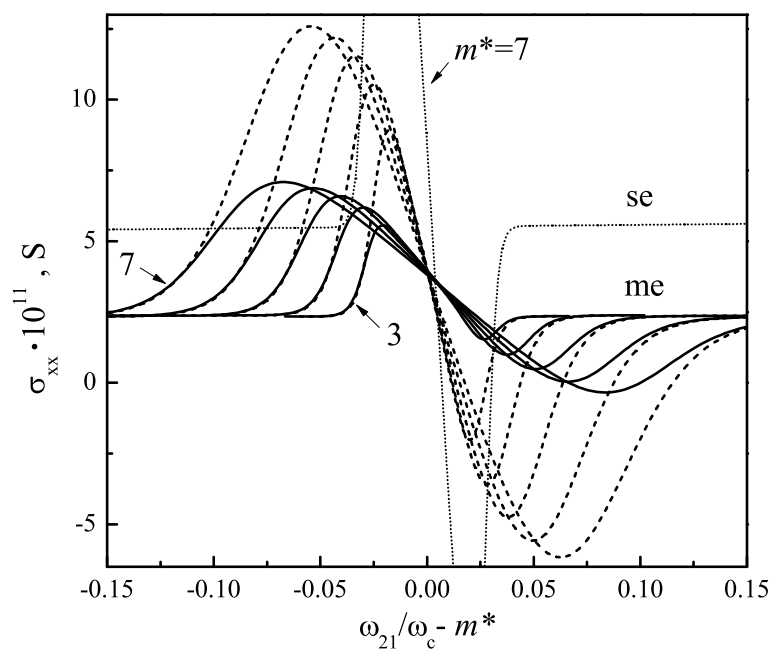

FIG. 5. Magnetoconductivity vs the parameter $\omega_{2,1} / \omega_{c}-m^{*}$ for $T=0.2 \mathrm{~K}$, and $n_{e}=10^{6} \mathrm{~cm}^{-2}$ : the single-electron treatment (dotted), the many-electron theory $\left[\Omega_{R}=2.5 \cdot 10^{7} \mathrm{~s}^{-1}\right.$ (solid), and $\Omega_{R}=5 \cdot 10^{7} \mathrm{~s}^{-1}$ (dashed)].

tremes of the many-electron lines (solid) from the point $\omega_{2,1} / \omega_{c}=m^{*}$ increases fast with $m^{*}$. For the comparison reason, the corresponding single-electron line (dotted) of $m^{*}=7$ is also plotted. It is interesting to note that under the assumption $T_{e}=T$, an increase in power (see dashed lines) reduces a little bit the distance of $\sigma_{x x}$ extremes from the level matching points. This behavior correlates with the behavior of corresponding $\bar{n}_{2}$ lines in Fig. 2. The electron heating induced by MW radiation acts in the opposite way, increasing the Coulomb broadening of $I_{l, n, l^{\prime} ; n^{\prime}}^{(\mathrm{me})}\left(\omega_{l, l^{\prime}}\right)$ (the frequency shift $x_{q} \Gamma_{C}^{2} / 4 T_{e}$ is independent of $T_{e}$ ) due to $\Gamma_{C} \propto \sqrt{T_{e}}$. Therefore, positions and broadening of the conductivity minima in an experiment can be used for estimation of electron temperature realized in this system in the presence of resonant radiation.

Positions of $\sigma_{x x}$ extremes depend also on $n_{e}$, as illustrated in Fig. 6 representing results obtained for two electron densities: $5 \cdot 10^{6} \mathrm{~cm}^{-2}$, and $2 \cdot 10^{7} \mathrm{~cm}^{-2}$. One can see differences in variations of conductivity minima and maxima. Conductivity minima definitely become more distant from the level matching points with the increase in $n_{e}$ for all $m^{*}$ presented in the figure. Regarding conductivity maxima, with the increase in $n_{e}$, they become more distant for three smallest level matching numbers $m^{*}=4,5$ and 6 , while for larger numbers $\left(m^{*}=8\right.$ and 9$)$ they become even closer to the level matching points. For the transition number $m_{c}^{*}=7$, the position of the corresponding maximum is practically unchanged. The $m_{c}^{*}$ becomes larger, if lower electron densities are compared. 


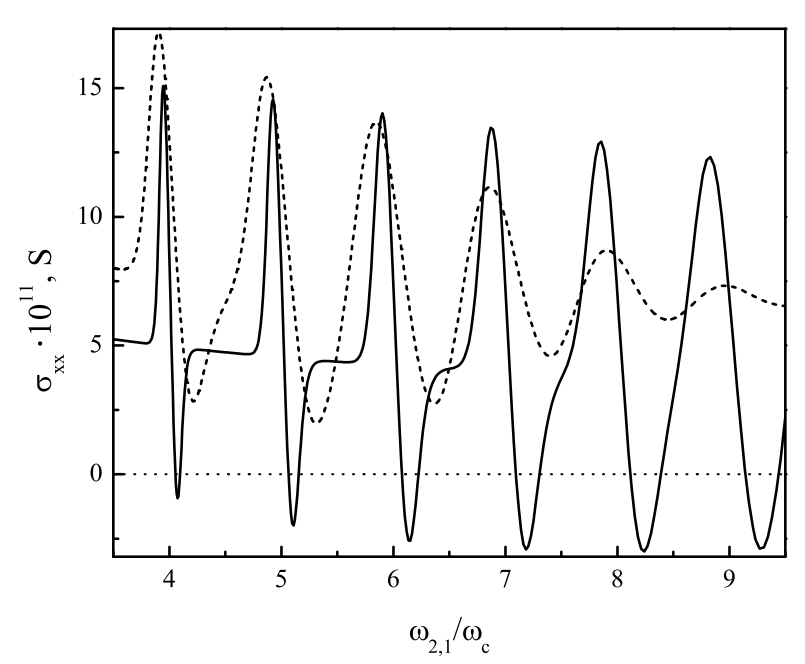

FIG. 6. Magnetoconductivity vs $\omega_{2,1} / \omega_{c}$ for $T=0.2 \mathrm{~K}$ and two electron densities: $n_{e}=5 \cdot 10^{6} \mathrm{~cm}^{-2}$ (solid) and $n_{e}=$ $2 \cdot 10^{7} \mathrm{~cm}^{-2}$ (dashed).

\section{DISCUSSION AND CONCLUSIONS}

The expression for the generalized dynamic factor given in Eqs. (15) and (16) together with equations for the effective collision frequency [Eqs. (20)-(22)] allows to describe photoconductivity of SEs on liquid helium in a wide range of the parameter $U_{C} / T$ up to the Wigner solid transition. The main conclusion of the many-electron theory reported in this work is that the unusual MWinduced magneto-oscillations of $\sigma_{x x}$ and ZRS can exist in multisubband 2D electron systems even under strong Coulomb coupling conditions $\left(U_{C} \gg T\right)$ relevant to experiments 2 .

Coulomb forces acting between electrons affect strongly the broadening of magneto-oscillations and positions of conductivity extremes, which become dependent on electron density, the level matching number $m^{*}$ and the electron temperature. Therefore, for SEs on liquid helium, in the many-electron theory, there are no fixed "magic" numbers describing positions of conductivity minima on the $\omega_{2,1} / \omega_{c}$-axis. The proximity of experimentally observed minima to $\omega / \omega_{c} \simeq m^{*}+1 / 4$, most likely, is accidental (valid only for a specific electron density). A substantial dependence of the distance of $\sigma_{x x}$ minima from the point $\omega / \omega_{c}=m^{*}$ on the level matching number $m^{*}$, which can be seen in experimental data of Ref. 2, agrees qualitatively with this conclusion.

Numerical calculations of SE magnetoconductivity shown in the previous Section were performed under the assumption that electrons are not substantially heated by the $\mathrm{MW}\left(T_{e} \simeq T\right)$. This assumption allows the manyelectron theory to reproduce remarkable shapes of $\sigma_{x x}$ oscillations observed for SEs on liquid helium. The inclusion of Coulomb interaction in the theory affects crucially the dependence of the amplitude of magnetooscillations on the magnetic field strength. In the many-electron theory, the amplitude of oscillations eventually begins decreasing with lowering $B$, which agrees with experimental observations. Even though the Coulombic effect suppresses amplitudes of magneto-oscillations, under experimental conditions of Ref. 2, the linear theory results in $\sigma_{x x}<0$ at certain ranges of magnetic and MW fields.

The comparison of theoretical and experimental $\sigma_{x x}(B)$ curves indicates that typically the real heating of SEs is not strong and most likely $T_{e} \sim T$. Since $\nu_{\text {intra }} \sim 1 / T_{e}\left(\Gamma_{l, n ; l^{\prime}, n^{\prime}}^{2}+x_{q} \Gamma_{C}^{2}\right)^{1 / 2}$, a strong increase in electron temperature $\left(T_{e} \gg T\right)$ would lead to deep drops of $\sigma_{x x}$ at the both sides of the level matching conditions, which contradicts to observations. In the single-electron theory, with an increase in electron temperature the signchanging correction $\nu_{\mathrm{A}}$ becomes more efficient than $\nu_{\text {intra }}$ and $\nu_{\mathrm{N}}$, because $\Gamma_{l, n}$ is independent of $T_{e}$. In the manyelectron theory, the Coulomb correction to the broadening of $S_{l, l^{\prime}}^{(\mathrm{me})}\left(q, \omega_{l, l^{\prime}}\right)$ increases with electron temperature, $\sqrt{x_{q}} \Gamma_{C} \propto T_{e}^{1 / 2}$, and the relative increase in efficiency of $\nu_{\mathrm{A}}$ as compared to $\nu_{\text {intra }}$ and $\nu_{\mathrm{N}}$ approaches a saturation, when $\sqrt{x_{q}} \Gamma_{C} \gg \Gamma_{l, n ; l^{\prime}, n^{\prime}}$. The electron heating affects positions of $\sigma_{x x}$ extremes, which also can be used for estimation of $T_{e}$. The many-electron theory of cold SEs results in minima distances from the level matching points which are of the same order, as those observed experimentally. This also agrees with the estimation $T_{e} \sim T$ given above.

Regarding negative conductivity values obtained in the linear theory, regions with $\sigma_{x x}<0$ are surely unstable, because any electron density fluctuation produces an electric field which increases the fluctuation. For the Coulomb liquid formed on the liquid helium surface, even the fluctuational field $\mathbf{E}_{f}^{(i)}$ can be a cause for instability, because excited SEs will be scattered against the restoring force $-e \mathbf{E}_{f}^{(i)}$. The edge of the $2 \mathrm{D}$ electron system on liquid helium is usually fixed by application of a dc confining field of Corbino and guard electrodes which also can be a cause for instability of the system at $\sigma_{x x}<0$.

A stable state can be achieved under the nonlinear transport regime by forming a strong steady current $j_{0}$, of which $\sigma_{x x}\left(j_{0}\right)=0$. In the $2 \mathrm{D}$ Coulomb liquid on the surface of liquid helium, formation of current domains is unlikely because of strong electron correlations. The SEs usually have no source and drain electrodes. Therefore, a steady current can be formed only by electrons circling the center of the electron pool. Since it is impossible to create a strong current density in the center, electrons will move to the edges of the electron liquid depleting the center. As a result, a nonuniform electron density distribution along the surface will be formed to provide radial electric field and a circling current, strong enough to make $\sigma_{x x}=0$. At a fixed magnetic field, a change in electron density additionally helps the system to leave the unstable regime, due to the many-electron effect described here. 
Thus, under the condition $\sigma_{x x}<0$ of the linear transport regime, electron moving against the confining force of Corbino and guard electrodes is the most likely way to reach a stable state. This scenario agrees with recent observations of the resonant photovoltaic effect for SEs on liquid helium ${ }^{11}$, where MW excitation causes a strong displacement of surface electrons towards the edges (against the confining force), if the magnetic field is close to the minima of the conductivity oscillations. Therefore, experimental observation of the strong density dependence of positions of conductivity minima consistent with the results of the many-electron theory would be an additional evidence for the negative conductivity as the origin of the resonant photovoltaic effect.

\section{APPENDIX}

According to definitions given in Eqs. (6)-(8), for the two-subband model $(l=1,2)$, the functions $w_{l, l^{\prime}}(y)$, describing electron-ripplon coupling, can be written as

$w_{l, l^{\prime}}(y)=\frac{A_{l} A_{l^{\prime}}}{4 \bar{\gamma}_{l, l^{\prime}}^{3}} \int_{0}^{\infty} e^{-x}\left[\frac{1}{y}-\frac{x}{\sqrt{y}} K_{1}(x \sqrt{y})\right] \lambda_{l, l^{\prime}}(x) d x$ where $\lambda_{1,1}=1$, and

$$
\lambda_{2,1}(x)=\left(1-\frac{1}{3} x\right), \quad \lambda_{2,2}(x)=\left[1-\frac{\bar{\gamma}_{2,1}}{3 \gamma_{2}} x\right]^{2} .
$$

The integrals of the right part of Eq. (26) can be found analytically, and we have $w_{1,1}(y)=w_{1}(y)$,

$$
\begin{gathered}
w_{2,1}(y)=\frac{A_{2} A_{1}}{4 \bar{\gamma}_{2,1}^{3}}\left[w_{1}(y)-\frac{1}{3} w_{2}(y)\right], \\
w_{2,2}(y)=\frac{A_{2}^{2}}{4 \gamma_{2}^{3}}\left[w_{1}(y)-\frac{2 \bar{\gamma}_{2,1}}{3 \gamma_{2}} w_{2}(y)+\left(\frac{\bar{\gamma}_{2,1}}{3 \gamma_{2}}\right)^{2} w_{3}(y)\right] .
\end{gathered}
$$

Here we use the following notations

$$
w_{1}(y)=-\frac{1}{1-y}+\frac{1}{(1-y)^{3 / 2}} \ln \left[\frac{1+\sqrt{1-y}}{\sqrt{y}}\right],
$$

$$
w_{2}(y)=-\frac{4-y}{(1-y)^{2}}+\frac{3}{(1-y)^{5 / 2}} \ln \left[\frac{1+\sqrt{1-y}}{\sqrt{y}}\right]
$$

$w_{3}(y)=-\frac{19-6 y+2 y^{2}}{(1-y)^{3}}+\frac{(12+3 y)}{(1-y)^{7 / 2}} \ln \left[\frac{1+\sqrt{1-y}}{\sqrt{y}}\right]$.

The analytical presentation of $w_{l, l^{\prime}}(y)$ given above is very useful for numerical evaluations of $\sigma_{x x}$.
1 D. Konstantinov and K. Kono, Phys. Rev. Lett. 103, 266808 (2009).

2 D. Konstantinov and K. Kono, Phys. Rev. Lett. 105, 226801 (2010)

3 M.A. Zudov, R.R. Du, J.A. Simmons, and J.R. Reno, Phys. Rev. B 64, 201311(R) (2001).

4 R. Mani, J.H. Smet, K. von Klitzing, V. Narayanamurti, W.B. Johnson, and V. Umansky, Nature 420, 646 (2002).

5 M.A. Zudov, R.R. Du, L.N. Pfeiffer, and K.W. West, Phys. Rev. Lett. 90, 046807 (2003).

6 A.V. Andreev, I.L. Aleiner, and A.J. Millis, Phys. Rev. Lett., 91, 056803 (2003).

7 V. I. Ryzhii, Fiz. Tverd. Tela 11, 2577 (1969) [Sov. Phys. Solid State 11, 2078 (1970)]; V.I. Ryzhii, R.A. Suris, and B. S. Shchamkhalova, Fiz. Tekh. Poluprovodn. 20, 2078 (1986) [Sov. Phys. Semicond. 20, 1299 (1986)].

8 A.C. Durst, S. Sachdev, N. Read, and S.M. Girvin, Phys. Rev. Lett. 91, 086803 (2003)

${ }^{9}$ Yu.P. Monarkha, Fiz. Nizk. Temp. 37, 108 (2011) [Low Temp. Phys. 37, 90 (2011)].

10 Yu.P. Monarkha, Fiz. Nizk. Temp. 37, 829 (2011) [Low Temp. Phys. 37, 655 (2011)].

11 D. Konstantinov, A.D. Chepelianskii, and K. Kono, arXiv:1101.5667 1 [cond-mat.mes-hall].
12 M.I. Dykman and L.S. Khazan, Zh. Eksp. Teor. Fiz. 77, 1488 (1979) [Sov. Phys. JETP 50, 747 (1979)].

13 Yu.P. Monarkha, E. Teske, and P. Wyder, Phys. Rep. 370, No. 1, pp. 1-61 (2002).

14 Yu.P. Monarkha and K. Kono, Two-Dimensional Coulomb Liquids and Solids, Springer-Verlag, Berlin Heildelberg (2004).

15 C. Grimes and G. Adams, Phys. Rev. Lett., 42, 795 (1979).

16 C.L. Zipfel, T.R. Brown, and C.C. Grimes, Phys. Rev. Lett. 37, 1760 (1976)

17 M.J. Lea, P. Fozooni, A. Kristensen, P.J. Richardson, K. Djerfi, M.I. Dykman, C. Fang-Yen, and A. Blackburn, Phys. Rev. B 55, 16280 (1997)

18 C. Fang-Yen, M.I. Dykman, and M.J. Lea, Phys. Rev. B 55, 16272 (1997)

19 R. Kubo, S.J. Miyake, N. Hashitsume, Solid State Phys. 17, 269 (1965).

20 T. Ando and Y. Uemura, J. Phys. Soc. Jpn. 36, 959 (1974).

21 Yu.P. Monarkha, S.S. Sokolov, A.V. Smorodin, and N. Studart, Fiz. Nizk. Temp., 36, 711 (2010) [Low Temp. Phys. 36, 565 (2010)].

22 Yu.P. Monarkha, Fiz. Nizk. Temp. 27, 627 (2001) [Low Temp. Phys. 27, 463 (2001)]. 